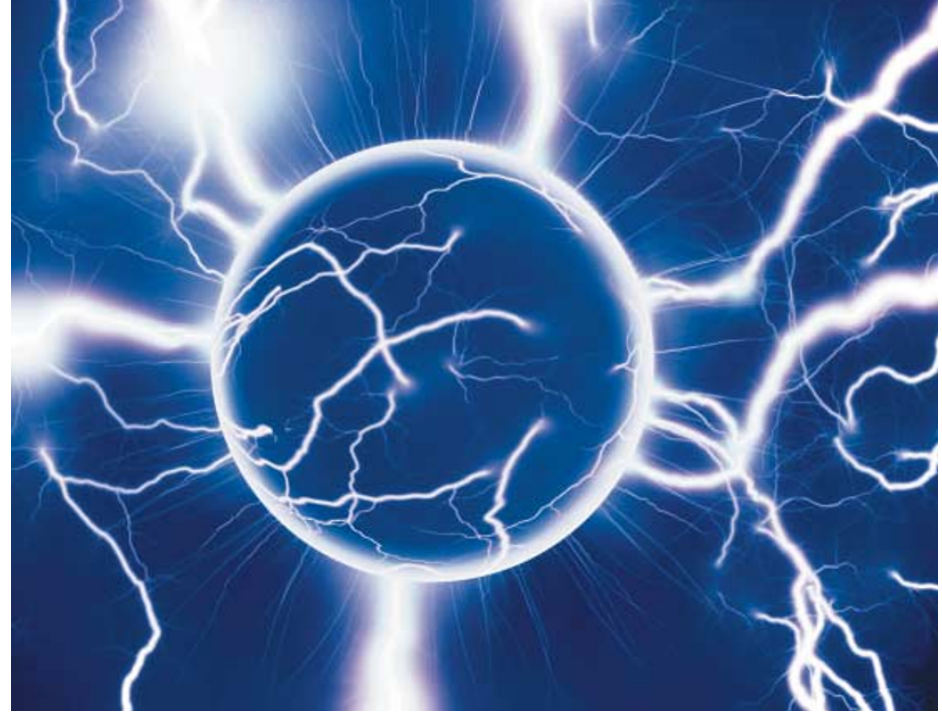

ANTIGEN PRESENTATION

\title{
Presenting a newly charged antigen
}

Peptide fragments presented in the context of MHC class II molecules are the typical antigens recognized by $\mathrm{CD} 4^{+} \mathrm{T}$ cells that express $\alpha \beta-\mathrm{T}$-cell receptors. However, a paper published in Cell now reports that certain polysaccharides, when processed by antigen-presenting cells (APCs), can associate with MHC class II molecules and induce $\mathrm{CD}^{+}$T-cell responses.

Previous studies have shown that in rodent models of intraabdominal abscess formation, abscesses form as a result of $\mathrm{CD} 4^{+}$ T-cell activation by bacterial capsular polysaccharides that contain a repeating zwitterionic-charge motif — a repeating unit containing both positive and negative charges. As zwitterionic polysaccharide (ZPS)induced activation of $\mathrm{CD} 4^{+} \mathrm{T}$ cells in vitro requires APC uptake of $\mathrm{ZPS}$, as well as MHC class II molecules, Cobb et al. set out to investigate whether ZPS uses the conventional MHC class II antigen-presentation pathway.

Using confocal microscopy, polysaccharide A (PS-A) — a Bacteroides fragilis ZPS - was observed on the cell membrane and within APCs. In both locations, PS-A colocalized with MHC class II molecules. Uptake of PS-A by APCs was mediated by endocytosis, and colocalization of PS-A with MHC class II molecules involved fusion of exocytic vesicles with the endosomes, both characteristics of the conventional MHC class II antigen-presentation pathway.
Analysis of PS-A taken up by APCs indicated that the polysaccharide was catabolized after overnight culture. When studied in vitro, PS-A processing was only achieved by oxidizing agents such as $\mathrm{O}_{3}$. Oxidation was also shown to be important in vivo, because compared with wildtype animals, markedly fewer mice lacking inducible nitric oxide synthase (iNOS) developed abscesses when challenged with $B$. fragilis or PS-A in adjuvant. Further evidence that processed PS-A fragments bound to MHC class II molecules are responsible for $\mathrm{CD} 4^{+} \mathrm{T}$-cell activation and abscess formation was provided by the observation that after overnight culture with PS-A, fragments of the ZPS could be detected bound to MHC class II molecules from wild-type APCs but not iNOS-deficient APCs.

This study, indicating that iNOSdependent processing of ZPS antigens yields carbohydrate fragments that associate with MHC class II molecules and induce $\mathrm{CD} 4^{+} \mathrm{T}$-cell responses, markedly increases the repertoire of pathogenic molecules that can stimulate $\mathrm{T}$ cells. The authors suggest that these observations might explain why some polysaccharide vaccines work so well and might provide new approaches to the design of vaccines for infectious diseases.

(2) References and links ORIGINAL RESEARCH PAPER Cobb, B. A., Wang, Q., Tzianabos, A. O. \& Kasper, D. L. Polysaccharide processing and presentation by the MHCIl pathway. Cell 117, 677-687 (2004).
RE SEARCH H I G H L I H TS

\section{IN BRIEF}

\section{INNATE IMMUNITY}

(2)

Toll-like receptor 9-dependent and -independent dendritic cell activation by chromatin-immunoglobulin $\mathrm{G}$ complexes.

Boulé, M. W. et al. J. Exp. Med. 14 June 2004 (doi:10.1084/jem20031942).

B-cell-receptor-mediated uptake of immune complexes containing chromatin and chromatin-specific IgG (chromatin-ICs) enables chromatin to trigger Toll-like receptor 9 (TLR9) and activate autoreactive B cells. Boulé et al. show that a similar two-receptor process can activate bone-marrow-derived dendritic cells (BMDCs): the production of tumour-necrosis factor in response to chromatin-ICs was impaired in TLR9- and MyD88-deficient BMDCs and was abolished in the absence of Fc $\gamma$ RIII. By contrast, production of the B-cell survival factor BAFF was TLR9 independent. This identification of two distinct chromatin-IC activation pathways in BMDCs might provide insight into the mechanisms of systemic lupus erythematosus pathogenesis.

\section{LYMPHOCYTE SIGNALLING}

Requirement for Tec kinases in chemokine-induced migration and activation of Cdc42 and Rac.

Takesono, A. et al. Curr. Biol. 14, 917-922 (2004).

The tyrosine kinases that regulate chemokine-induced signalling pathways were ill-defined until this report identified a role for the TEC kinases ITK and RLK. Stromal-cell-derived factor $1 \alpha$ (SDF1 $\alpha$ ) induced tyrosine phosphorylation of ITK and RLK, and a dominant-negative ITK mutant decreased migration in response to this chemokine. Impaired migration was associated with decreased actin polymerization and decreased activation of the actin-cytoskeleton regulators CDC42 and RAC. T cells from $I t k^{-l-} R l k^{-/-}$mice also showed decreased migration in response to SDF1 $\alpha$. These TEC kinases also regulate actin polymerization induced by T-cell receptor (TCR) stimulation, so the authors suggest that chemokine-receptor and TCR-signalling pathways converge to induce actin-cytoskeleton reorganization.

\section{HAEMATOPOIESIS}

\section{Stepwise reprogramming of B cells into macrophages.}

Xie, H. et al. Cell 117, 663-676 (2004).

This study looked at the transcription-factor networks that determine whether a multipotent haematopoietic progenitor will enter the lymphoid or myeloid lineages. When the myeloid transcription factors $\mathrm{C} / \mathrm{EBP}-\alpha$ or $\mathrm{C} / \mathrm{EBP}-\beta$ are expressed in $\mathrm{B}$-cell precursors or mature splenic $B$ cells, these cells are reprogrammed to become functional macrophages. This was the result of both downregulation of the late B-cell-specific marker CD19, through inhibition of the B-cell commitment factor PAX5, and upregulation of MAC1 and other myeloid markers in synergy with endogenous PU.1. This antagonism between C/EBP and PAX5 is a novel mechanism of lineage commitment, and the authors suggest that the decision to become a B cell or macrophage depends on which becomes dominant. This ties in with the fact that the B-cell to macrophage conversion frequency depended on the dose of C/EBP. 